\title{
Electroencephalographic Abnormalities in First Onset Afebrile and Complex Febrile Seizures and Its Association with Type of Seizures
}

\author{
Authors \\ Dr Mohammed Ashfaque Tinmaswala ${ }^{1}$, Dr Valinjker S.K ${ }^{2}$, Dr Shilpa Hegde \\ Dr Parmeshwar Taware ${ }^{4}$ \\ ${ }^{1,3}$ Senior Residents, ${ }^{2}$ Associate Professor, ${ }^{4}$ Assistant Professor
}

Department of Pediatrics, Grant Government Medical College and JJ Group of Hospitals Mumbai

Corresponding Author

Dr Mohammed Ashfaque Tinmaswala

Senior Resident, Grant Government Medical College and JJ Group Of Hospital Mumbai

Email:dr.ashfaq.memon@gmail.com, Mobile no: 7276738362

\begin{abstract}
:
Aims and Objectives: (1) To determine the frequency of abnormal Electroencephalogram in children presenting with first-onset afebrile and complex febrile seizures. (2) To find out most common EEG patterns associated with first-onset afebrile and complex febrile seizures.
\end{abstract}

Study Design:- It was an observational prospective, stratified randomized cohort study done in a tertiary care hospital in a metropolitan city.

Materials and Methods:- The study was approved by the Institutional ethical committee. The patients attending the Emergency, in-patient and outpatient Departments of Pediatrics Division of ourinstitute were enrolled for the study as per the criteria given. It was a prospective, stratified-randomized cohort study conducted on children in the age group of 6 months to 12 years having first-onset unprovoked seizures and Complex febrile seizures.

Results:- The mean age of seizure occurrence was 6.3 years. Male preponderance was noticed with a ratio of 1.63:1.EEG abnormalities were seen jn 40 patients (40\%). EEG abnormalities were most commonly associated with partial seizures (53.57\%) followed by generalized seizures (40\%). EEG abnormalities were least commonly seen with complex febrile seizures (21.85\%). Sharp and spike waves, alone or in the combination were overall most common EEG findings. In generalized seizures, most common EEG abnormality found was sharp waves which were seen in 8/18(44.44\%). Second most common pattern of EEG abnormality in generalized seizures were either spike waves 3/18(16.67\%) or sharp and spike waves 3/18(16.67\%). Asymmetry with sharp waves and abnormal background with sharp waves are seen in $2 / 18$ (11.11\%) patients each. In partial seizures, most common pattern of EEG abnormality was spike waves seen in 4/15 (26.67\%) second most common EEG abnormality in partial seizures was abnormal background with sharp waves and spikes which was seen in 3/15(20\%).sharp waves, sharp and spike waves and asymmetry with sharp and spike waves. Incidence of each of these EEG abnormalities was same I e 2/15 (13.33) while asymmetry with sharp waves was seen in 1 patient (6.67\%). In complex febrile seizures Asymmetry with sharp waves and spike, abnormal background with sharp waves and asymmetry with sharp waves were most common EEG abnormalities seen in 4/7(57.14\%), 2/7(28.57) and $1 / 7(14.29 \%)$ patients respectively.

Conclusion: EEG, preferably 3-4 days after the seizures, should be done in all pediatric patients presenting either with first onset afebrile or complex febrile seizures.

Keywords: Electroencephalogram, First onset afebrile seizures, complex febrile seizures 


\section{INTRODUCTION}

Seizures are the most common pediatric neurological disorder. 4-10\% percent of children suffer at least one episode of seizure in the first 16 years of life. The incidence is highest in children less than 3 years of age, with a decreasing frequency in older children ${ }^{[1]}$. Though not available everywhere EEG and neuroimaging are vital to the management of epilepsy. EEG is recommended as a part of the neurodiagnostic evaluation of the child with an apparent unprovoked seizure ${ }^{[2]}$. Well appearing children who have experienced first-time afebrile seizure should be referred for outpatient EEG testing ${ }^{[3,4]}$. It is important to note that a normal EEG does not rule out epilepsy or other underlying neurologic disorder but an abnormal EEG increases the chances of later development of epilepsy ${ }^{[5] .}$ Also the appropriate evaluation of children presenting with seizures is sometimes impossible without an EEG. Another important aspect of using EEG is that the seizure and epileptic syndrome classifycations are based upon combined clinico electroencephalographic manifestations. Many epileptic syndromes were mainly identified because of their EEG manifestations. EEG is also valuable in differentiating generalized and focal epilepsies.

The guidelines for EEG and Neuroimaging after first episode of afebrile seizures and complex febrile seizures are not uniform. EEG is not always used appropriately in epilepsy care; evidence-based guidance on its role is limited by a paucity of high quality data and methodological deficiencies in published studies ${ }^{[6]}$. Moreover though being a noninvasive test it's considered to be entirely safe there are chances that improper technique, ignorance about the phenomenon of artifacts and technical faults may turn this investigation modality into a harmful investigation sometimes leading to unnecessary treatment. Other facts worth considering are that demonstration of epileptiform abnormalities in the EEG does not in itself means that the patient is having epilepsy or indicate that the patient has a seizure disorder. Also non epileptic individuals sometimes exhibits epileptogenic abnormalities in their EEGs. A large study of standard EEGs in healthy mostly male adults with no declared history of seizures showed epileptiform discharge in $0.5 \%$ of the subjects ${ }^{[7] . ~ A ~ s l i g h t l y ~ h i g h e r ~}$ incidence of $2-34 \%$ is found in healthy children and in non-epileptic patients seen in hospital EEG clinics ${ }^{[8]}$. Thus, great caution is necessary when assessing the significance of epileptiform activity in such circumstances, particularly if the history offers little or no indication that the patient has epilepsy on clinical grounds. It's especially difficult to subject all the patients presenting with first onset afebrile and complex febrile seizures to investigations like EEG and Neuroimaging in a developing country like India where resources are scarce ${ }^{[9]}$. It's also a well-known fact that as a pediatrician many are perplexed with the extent one needs to go for investigating these children who present with first onset afebrile and complex febrile seizures. Unnecessary investigations without a careful history and through clinical examination might only serve to confuse the issue of diagnosis and may have detrimental effects on the patients trust in the doctor and his management plan ${ }^{[9] .}$

\section{MATERIALS AND METHODS}

The patients attending the Emergency, in-patient and outpatient Departments of Pediatrics Division of this institute were enrolled for the study as per the criteria given. It was a prospective, stratifiedrandomized cohort study conducted on children in the age group of 6 months to 12 years having firstonset unprovoked seizures and Complex febrile seizures. The children below 6 months were excluded because the causes of seizures in that age group is different. Children were also excluded from the study if the seizure resulted from an acute situational etiology such as toxin or trauma. They were also excluded if they had a chronic neurologic illness limiting their activities of daily living, such as cerebral palsy, mental retardation, and pervasive development disorders, or had other abnormalities on neurologic examination or had simple febrile seizure. Parents 
of the patients signed consent forms before participation. A detailed clinical and developmental history was taken and physical and neurological examination was carried out to identify a predominant seizure type and potential epilepsy syndrome. A neurological examination was defined as abnormal when there was any focal neurological abnormality. Seizure type was classified on the basis of "The International League against Epilepsy classification" Blood samples of all subjects were drawn on admission and routine laboratory studies were performed. Complete blood counts, blood sugar, serum $\mathrm{Na}+$, $\mathrm{K}+$, calcium were analyzed in order to exclude possible metabolic disorder, An EEG was performed in all the subjects in the study. EEG was done after at least 4 days and not later than 2 weeks of the first episode of afebrile or complex febrile seizures. This time period was decided keeping in mind the fact that an early EEG may show some abnormalities such as postictal slowing which may be transient. Also this was in conformity with the recommendations of Expert committee on pediatric epilepsy - Indian academy of pediatrics which recommended that after an unprovoked seizure the EEG should be done not earlier than 3-4 days of last episode of seizures. EEG was using 21-channel EEG machine (Model EE 18) from recorder and Medicare system. A total of 22 electrodes were placed using children montage. EEG was performed within 2 weeks of first episode of seizures. The EEG was analyzed by a clinical neurologist from the Department of Neurology of our institute. EEG data was classified for the presence or absence of (1) overall abnormal EEG (2) epileptiform discharges (3) slowing of waves (4) asymmetry (5) abnormal background. Epileptiform activity was also further differentiated on the basis of it being focal or generalized. Qualitative data was represented in form of frequency and percentage. Association between qualitative variables was assessed by Chi-Square test with Continuity Correction for all $2 \times 2$ tables and Fisher's exact test for all $2 \times 2$ tables where $\mathrm{p}$-value of Chi-Square test was not valid due to small counts. Adjacent row data of more than 2X2 tables was pooled and Chi-Square test reapplied in case more than $20.0 \%$ cells having expected count less than 5. Quantitative data was represented using mean \pm sd and Median \& IQR (Interquartile range). Diagnostic Efficacy of EEG was assessed by calculating Sensitivity, Specificity, Positive Predictive Value, Negative Predictive Value, and Positive Likelihood Ratio \& Negative Likelihood Ratio. Results were graphically represented where deemed necessary. SPSS Version 17 was used for analysis.

\section{OBSERVATIONS}

Hundred children aged between 6 months to 12 years presenting with either first onset afebrile seizures or first onset complex febrile seizures in a tertiary care hospital in a metro were enrolled

Table 1 : Type of seizures as per the age of patients

\begin{tabular}{|l|l|l|l|l|l|}
\hline \multirow{2}{*}{$\begin{array}{l}\text { Age } \\
(\text { years })\end{array}$} & & \multicolumn{2}{|l|}{ Type of seizures } & \multirow{2}{*}{ Total } \\
\cline { 3 - 6 } & & Complex Febrile & Generalized & Partial & \\
\hline \multirow{2}{*}{$\mathbf{6}$} & No. & 22 & 11 & 3 & 36 \\
\cline { 2 - 6 } & $\mathbf{\%}$ & $61.1 \%$ & $30.6 \%$ & $8.3 \%$ & $100.0 \%$ \\
\hline \multirow{2}{*}{$\mathbf{6}$} & No. & 10 & 29 & 25 & 64 \\
\cline { 2 - 6 } & $\%$ & $15.6 \%$ & $45.3 \%$ & $39.1 \%$ & $100.0 \%$ \\
\hline \multirow{2}{*}{ Total } & No. & 32 & 40 & 28 & 100 \\
\cline { 2 - 6 } & $\%$ & $32.0 \%$ & $40.0 \%$ & $28.0 \%$ & $100.0 \%$ \\
\hline
\end{tabular}




\begin{tabular}{|l|l|l|l|l|}
\hline Chi-Square Tests & Value & df & p-value & Association is- \\
\hline Pearson Chi-Square & 23.921 & 2 & $6.39 \mathrm{E}-06$ & Significant \\
\hline
\end{tabular}

Patients were divided into two groups on the basis of age. Group one was constituted by children aged 6 months to 6 years and included 36/100 (36) patients. Group two was constituted by children $>6$ years to 12 years old and included 64/100 $(64 \%)$ patients. In the age group of 6 months to $\leq 6$ years, 22/36 (61.11\%) had CFS and 11/36 $(30.56 \%)$ had generalized seizures and 3/36(8.33\%) had partial seizures. Chi square test was applied and was found to be Significant. Among children $>6$ years to 12 years old, partial seizure was seen in 25/64 (39.06\%), generalized seizure in $29 / 64(45.31 \%)$ and only $10 / 64$ (15.63\%) had CFS. In the age group from 6 months to 6 years most common form of seizures were complex febrile and least common seizures were partial seizures. Whereas 6years onwards the most common form of seizures were generalized seizures while complex febrile seizures were least common in this age group. When X2 test was applied for Age distribution and type of seizures the difference was found to be "significant".

Table 2: Association of sex distribution and type of seizures amongst the studied cases.

\begin{tabular}{|l|l|l|l|l|l|}
\hline \multirow{2}{*}{ Sex } & \multicolumn{2}{|l|}{ Type of seizures } & \multirow{2}{*}{ Total } \\
\cline { 3 - 6 } & & Complex Febrile & Generalized & Partial & \\
\hline \multirow{2}{*}{ Female } & No. & 15 & 14 & 9 & 38 \\
\cline { 2 - 6 } & $\%$ & $39.5 \%$ & $36.8 \%$ & $23.7 \%$ & $100.0 \%$ \\
\hline \multirow{2}{*}{ Male } & No. & 17 & 26 & 19 & 62 \\
\cline { 2 - 6 } & $\%$ & $27.4 \%$ & $41.9 \%$ & $30.6 \%$ & $100.0 \%$ \\
\hline \multirow{2}{*}{ Total } & No. & 32 & 40 & 28 & 100 \\
\cline { 2 - 6 } & $\%$ & $32.0 \%$ & $40.0 \%$ & $28.0 \%$ & $100.0 \%$ \\
\hline
\end{tabular}

\begin{tabular}{|l|l|l|l|l|}
\hline Chi-Square Tests & Value & df & p-value & Association is- \\
\hline $\begin{array}{l}\text { Pearson Chi- } \\
\text { Square }\end{array}$ & 1.630 & 2 & 0.443 & Not significant \\
\hline
\end{tabular}

Out of 100 cases 62 were males and 38 were Females. Amongst 62 Males generalized seizures were seen in $26(26 \%)$ patients Partial seizures were seen in 19 (19\%) patients and complex febrile seizures were seen in $17(17 \%)$ patients. Amongst 38 female patients 14(14\%) had generalized seizures $9(9 \%)$ had partial seizures and $15(15 \%)$ had complex febrile seizures. When $\mathrm{X} 2$ test was applied for sex distribution and type of seizures the test was found to be "Not significant". 
Table 3: Types of seizures amongst the studied cases.

\begin{tabular}{|l|l|l|l|}
\hline Type Of Seizures & Subtype & No of cases & percentage \\
\hline \multirow{5}{*}{ Generalized Seizures } & Absence & 6 & $15 \%$ \\
\cline { 2 - 4 } & Tonic Clonic & 32 & $80 \%$ \\
\cline { 2 - 4 } & Myoclonic & 2 & $5 \%$ \\
\cline { 2 - 4 } & Total & 40 & $40 \%$ \\
\hline \multirow{5}{*}{ Partial seizures } & simple & 6 & $21.42 \%$ \\
\cline { 2 - 4 } & complex & 14 & $50 \%$ \\
\cline { 2 - 4 } & secondary generalized & 8 & $28.57 \%$ \\
\cline { 2 - 4 } & Total & 28 & $28 \%$ \\
\hline Complex febrile seizures & 32 & $32 \%$ \\
\hline Total & 100 & $100 \%$ \\
\hline
\end{tabular}

The most common type of seizures observed In cases were generalized 40/100 (40\%) followed by Complex febrile seizures 32/100 (32\%), Partial Seizures were seen in $28 / 100(28 \%)$. Out of generalized seizures tonic clonic seizures were seen in 32 cases $(32 \%)$ absence and myoclonic seizures were seen in $6(6 \%)$ and $2(2 \%)$ patients. Amongst partial seizures complex partial seizures were seen in 14 (14\% cases) followed by partial seizures with secondary generalization which were seen in 8 ( $8 \%$ ) cases. Simple partial seizures were seen in $6(6 \%)$ cases. Overall most common form of seizures were Generalized Tonic Clonic (32\%) and least common seizures were myoclonic seizures which were seen in $2(2 \%)$ cases.

Table 4: EEG abnormalities in studied cases.

\begin{tabular}{|l|l|l|l|l|l|}
\hline \multirow{2}{*}{ EEG } & \multicolumn{2}{|l|}{ Type of seizures } & \multirow{2}{*}{ Total } \\
\cline { 3 - 6 } & & Complex Febrile & Generalized & Partial & \\
\hline \multirow{2}{*}{ Abnormal } & No. & 7 & 18 & 15 & 40 \\
\cline { 2 - 6 } & $\%$ & $21.9 \%$ & $45.0 \%$ & $53.6 \%$ & $40.0 \%$ \\
\hline \multirow{2}{*}{ Normal } & No. & 25 & 22 & 13 & 60 \\
\cline { 2 - 6 } & $\%$ & $78.1 \%$ & $55.0 \%$ & $46.4 \%$ & $60.0 \%$ \\
\hline \multirow{2}{*}{ Total } & No. & 32 & 40 & 28 & 100 \\
\cline { 2 - 6 } & $\%$ & $100.0 \%$ & $100.0 \%$ & $100.0 \%$ & $100.0 \%$ \\
\hline
\end{tabular}

\begin{tabular}{|l|l|l|l|l|}
\hline Chi-Square Tests & Value & df & p-value & Association is- \\
\hline Pearson Chi-Square & 6.946 & 2 & 0.031 & Significant \\
\hline
\end{tabular}

The Electroencephalographic abnormalities were overall found in $40 / 100(40 \%)$ patients. EEG abnormalities were most commonly associated with Partial seizures where 15/28 (53.5\%) had 


\section{JMSCR Vol.||03||Issue||08||Page 7073-7082||August}

abnormal EEG recordings. EEG abnormalities were least commonly seen with complex febrile seizures where $7 / 32(21.5 \%)$ patient had abnormal EEG. Patients with generalized seizures had abnormal EEG in 18 out of 40 patients (45\%).
When X2 test was applied for EEG abnormalities and type of seizures the association was found to be significant.

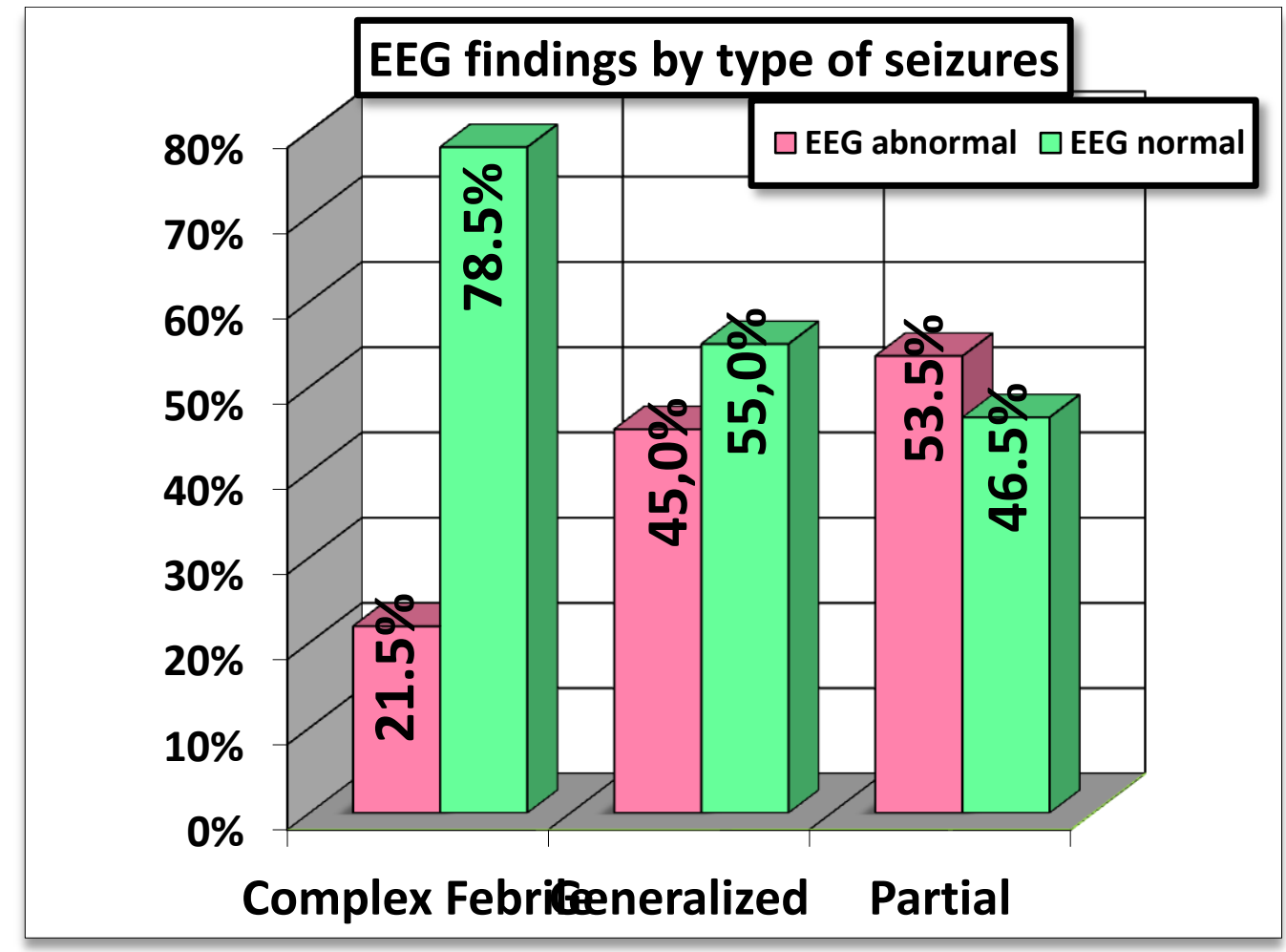

Fig 1 : EEG finding by Type of Seizures

Table 5: Type Of EEG Abnormalities in studied cases

\begin{tabular}{|c|c|c|c|c|c|c|}
\hline \multirow[t]{2}{*}{ Type Of EEG Abnormalities } & \multicolumn{2}{|c|}{ Generalized } & \multicolumn{2}{|c|}{ Partial } & \multicolumn{2}{|c|}{$\begin{array}{l}\text { Complex } \\
\text { Febrile }\end{array}$} \\
\hline & $\mathbf{N}$ & $\%$ & $\mathbf{N}$ & $\%$ & $\mathbf{N}$ & $\%$ \\
\hline Assymetry & $\mathbf{0}$ & $\mathbf{0}$ & 1 & $3.57 \%$ & $\mathbf{0}$ & $\mathbf{0}$ \\
\hline Sharp waves & 8 & $20 \%$ & 2 & $7.14 \%$ & $\mathbf{0}$ & $\mathbf{0}$ \\
\hline Spike Waves & 3 & $7.5 \%$ & 4 & $14.28 \%$ & $\mathbf{0}$ & $\mathbf{0}$ \\
\hline Assymetry with sharp waves & 2 & $5 \%$ & 1 & $3.57 \%$ & 1 & $3.1 \%$ \\
\hline Sharp and spike waves & 3 & $7.5 \%$ & 2 & $7.14 \%$ & $\mathbf{0}$ & $\mathbf{0}$ \\
\hline Assymetry with sharp waves and spikes & $\mathbf{0}$ & $\mathbf{0}$ & 2 & $7.14 \%$ & 4 & $12.5 \%$ \\
\hline
\end{tabular}




\begin{tabular}{|l|l|l|l|l|l|l|} 
Abnormal Background with sharp waves & 2 & $5 \%$ & 0 & 0 & 2 & $6.2 \%$ \\
\hline $\begin{array}{l}\text { Abnormal Background with sharp waves and } \\
\text { spikes }\end{array}$ & 0 & 0 & 3 & $10.71 \%$ & 0 & 0 \\
\hline Toal Abnormal EEGs & 18 & $45 \%$ & 15 & 53.57 & 7 & $21.85 \%$ \\
\hline Normal EEG & 22 & $55 \%$ & 13 & 46.42 & 25 & $78.2 \%$ \\
\hline Total EEGs & 40 & $100 \%$ & 28 & $100 \%$ & 32 & $100 \%$ \\
\hline
\end{tabular}

In generalized seizures, most common EEG abnormality found was sharp waves which were seen in 8/18(44.44\%). Second most common pattern of EEG abnormality in generalized seizures were either spike waves $3 / 18(16.67 \%$ ) or sharp and spike waves $3 / 18(16.67 \%)$. Asymmetry with sharp waves and abnormal background with sharp waves are seen in $2 / 18(11.11 \%)$ patients each. In partial seizures, most common pattern of EEG abnormality was spike waves seen in $4 / 15$ (26.67\%) second most common EEG abnormality in partial seizures was abnormal background with sharp waves and spikes which was seen in $3 / 15(20 \%)$.sharp waves, sharp and spike waves and asymmetry with sharp and spike waves. Incidence of each of these EEG abnormalities was same ie $2 / 15$ (13.33) while asymmetry with sharp waves was seen in 1 patient $(6.67 \%)$. In complex febrile seizures Asymmetry with sharp waves and spike, abnormal background with sharp waves and asymmetry with sharp waves were most common EEG abnormalities seen in 4/7(57.14\%), $2 / 7(28.57)$ and $1 / 7(14.29 \%)$ patients respectively.

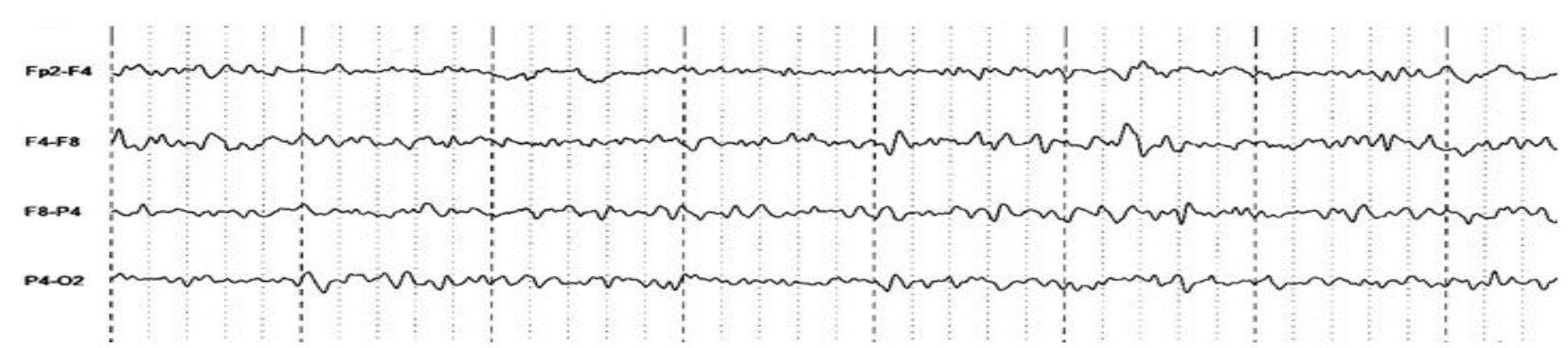

Fig 2: Most common EEG abnormality in generalized seizures were sharp waves

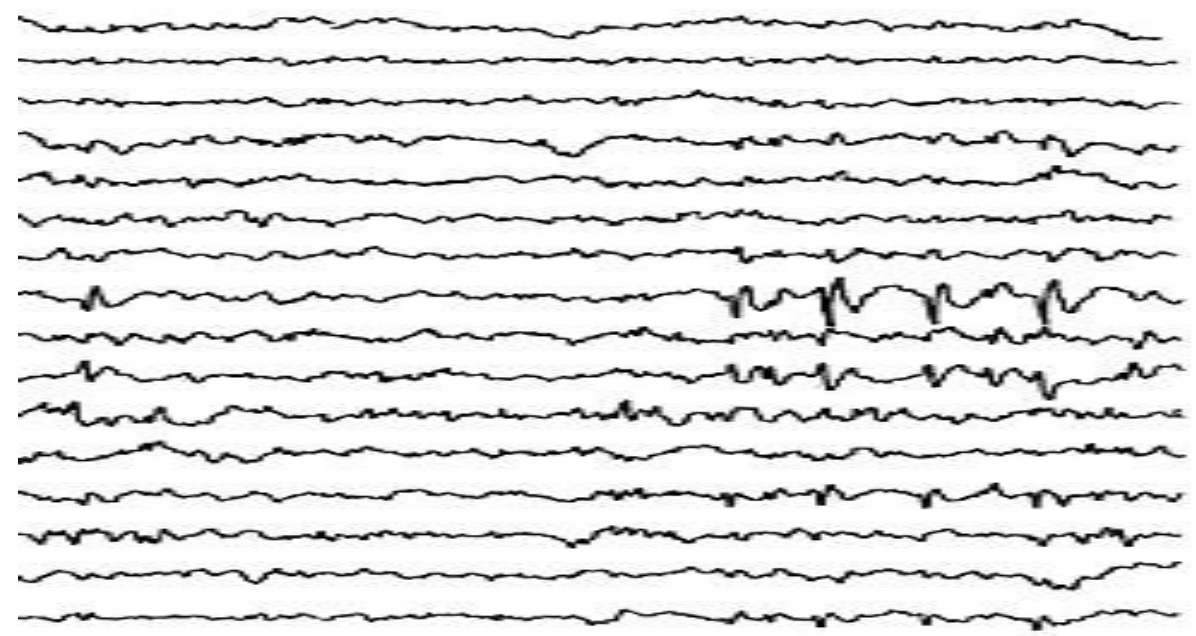

Fig 3 : Most common EEG abnormality in partial seizures were spike waves 


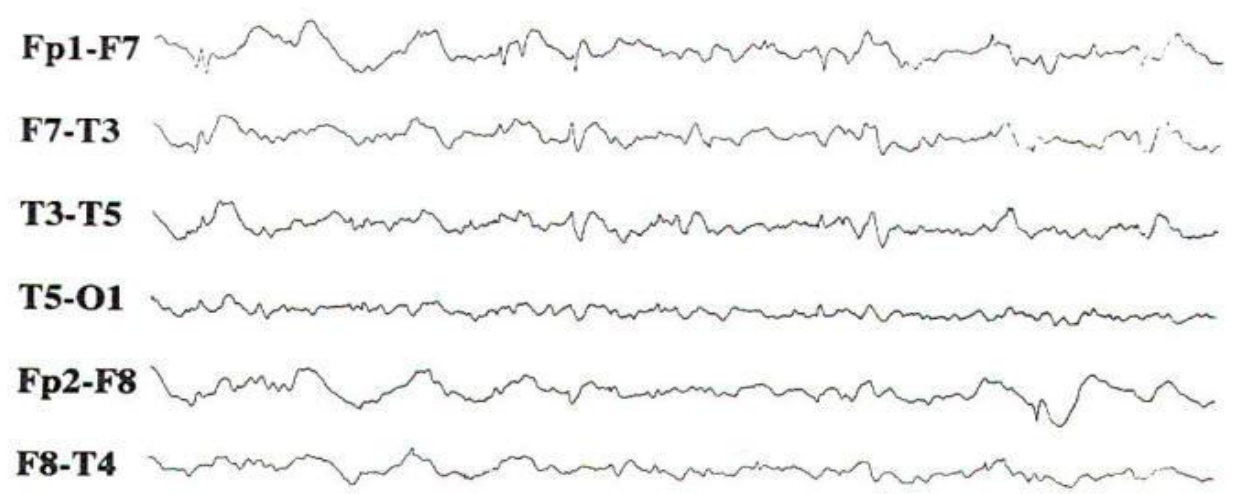

Fig 4: Assymetry with sharp waves and spikes was the most common EEg abnormality seen in complex febrile seizures

\section{DISCUSSION}

As a major portion of seizures occurring in children is due to febrile seizures (simple or complex) the patients in the study group were divided into 2 group first group consisted of those patients who presented with seizures from 6 months to 6 years and second group was from 6 years to 12 years. In first group (6m-6yrs) the most common type of seizures seen were complex febrile seizures $(61.11 \%)$. Generalized and partial seizures were seen in $11 \%$ and $3 \%$ cases respectively. In second group comprising of children in the age group of 6years to 12 years the most common type of seizure observed was generalized seizures $(45.31 \%)$ followed by partial $(39 \%)$ and complex febrile seizures which were seen in $15.62 \%$. In a similar study Akhterrasool et al ${ }^{[10]}$ found that in between $6 \mathrm{~m}$ $6 \mathrm{yr}$ the incidence of complex febrile seizures to be $55 \%$. And the incidence of generalized and partial seizures and of undetermined seizures to be $32 \%$, $8 \%$ and $5 \%$ respectively. Various types of seizures after 6yr observed were generalized, partial and complex seizures which were seen in $47.7 \%$, $44.3 \%$ and $5.1 \%$ patients respectively. In our study the most common type of seizures between 6 months to 6 years and 6 years onwards were similar to that of the study conducted by Rasool et al ${ }^{[10]}$. In this study of 100 cases 62 were males and 38 were females. Male preponderance was at par with studied conducted by rasool et al ${ }^{[10]}$, Simone carreiro et al ${ }^{[11]}$ and Maytal $\mathrm{J}^{[12]}$ who reported male preponderance with the incidence in males being $58.7 \%, 55.3 \%$ and $51.51 \%$.
In this study of 100 cases the most common form of first onset seizures was Generalized seizures which were seen in $40 \%$ patients followed by complex febrile seizures and partial seizures which were seen in $32 \%$ and $28 \%$ respectively. Amongst the generalized seizures the most common form of seizures seen was generalized tonic clonic seizures which were seen in 32/40 (80\%) patients. Amongst partial seizures complex partial seizures were the commonest form of seizures which were seen in 14/28 (50\%) patients . One of the objective of this study was to find out incidence of EEG abnormalities in first onset afebrile and complex febrile seizures. In this study EEG abnormalities were found in $40 \%$ patients (40/100). Shinnar et al [13] found EEG abnormalities in $42.05 \%$ patients in the study conducted by them. These findings were consistent with similar observations made and King et al [14]. They reported EEG abnormalities in $34 \%$ and $51 \%$ depending upon the time of EEG(less than 24 vs after $24 \mathrm{hrs}$ of seizures). The EEG abnormality of CFS group in our study was $15.6 \%$ (10/64). Similar observations were made by Charuta Joshi et al ${ }^{[15]}$. In case of first episode of complex febrile seizures EEG abnormalities were reported by Yucel o et al[16]to be $44.65 \%$ similar results were also reported by Rasool et al. The only study which reported completely different results was done by Maytal et al ${ }^{[12]}$ in this study EEG abnormalities found was $0 \%$. On analyzing individual abnormalities in EEG, our study observed that sharp wave and spikes (either alone or both) were the most common abnormality 
observed. In generalized seizures most common EEG abnormality found was sharp waves. Similarly spike waves and asymmetry with sharp waves and spikes were most common Abnormal EEG patterns seen in partial and complex febrile seizures respectively. EEG abnormalities are subject to many factors like time of EEG. Whether the EEG was sleep deprived or without sleep deprivation. King et al[14 ]reported that EEG is less likely to be abnormal if done late after episode of seizure. Sometimes they are over reported to be abnormal Benbadis et al [17] reported that over interpretation of a normal EEG as abnormal EEG was most commonly due to labelling of wicket spikes, hypnagogic hypersynchrony and hyperventilation induced slowing as epileptiform discharges.

\section{SUMMARY AND CONCLUSIONS}

A total of hundred children in age group of 6 months to 12 years with either first onset afebrile or complex febrile seizures comprised the study group.The mean age of seizure occurrence was 6.3 years. Male preponderance was noticed with a ratio of 1.63:1.Majority of the patients had motor symptoms. Generalized seizures $(40 \%)$ were the commonest type of seizures encountered followed by focal and complex febrile seizures which were seen in $28 \%$ and $32 \%$ respectively.Past history of seizures, meningitis and developmental delay being exclusion criteria were not present in any patient. Neurologic deficit (Todd's paralysis) was noticed in 4(4\%) patients at the time of admission but resolved in all of them before discharge. EEG abnormalities were seen jn 40/100(40\%) patients. EEG abnormalities were most commonly associated with partial seizures (53.57\%) followed by generalized seizures (40\%). EEG abnormalities were least commonly seen with complex febrile seizures $(21.85 \%)$.

From our findings we recommend that EEG should be done in all paediatric patients presenting with first onset afebrile or complex febrile seizures.

Conflict of interest: None

\section{REFERENCES}

1. Friedman MJ, Sharieff GQ: Seizures in children. PediatClin North Am 2006,53:257-277.

2. Hirtz D, Ashwal S, Berg A, Bettis D, Camfield C, Camfield $\mathrm{P}$, et al.Practice parameter: Evaluating a first nonfebrile seizure in children: Report of the quality standards subcommittee of the American Academy of Neurology, the Child Neurology Society, and the American Epilepsy Society. Neurology 2000;55:61623.

3. Shneker BF, Fountain NB. Epilepsy. Dis Mon 2003;49:426 - 78.

4. Reuter D, Brownstein D. Common emergent pediatric neurologic problems. Emerg Med Clin North Am 2002;20(1):155 - 76

5. Scheuer ML, Pedley TA. The evaluation and treatment of seizures. N Engl J Med 1990;323:1468 - 74

6. The diagnosis and management of the epilepsies in adults and children in primary and secondary care. National Institute for Clinical Excellence, October 2004

7. Gregory RP, Oates T, Merry RTG. EEG epileptiform abnormalities in candidates for aircrew training. Electroencephalogr ClinNeurophysiol 1993;86:75-77

8. Zifkin L, Ajmonemarsan C. Incidence and prognostic significance of 'epileptiform' activity in the EEG of non-epileptic subjects. Brain 1968;91:751-778

9. R A Mackenzie Ethical considerations in epilepsy management Medicine and law 23(4):781 (2004) PMID 15685916

10. AkhterRasool, Suhil A. Choh, Nisar A. Wani, et al Role of electroencephalogram and neuroimaging in first onset afebrile and complex febrile seizures in children from Kashmir J PediatrNeurosci. 2012 Jan-Apr; 7(1): 9-15.

11. Simone CV, Paulo Breno NL, Monica JS, Adriana BO, Alaides SFO, AlfredoLJ.First unprovoked seizure: Clinical and 
electrographic aspects. J Epilepsy Clin Neurophysiol 2006;12:69-72

12. Maytal J, Krauss JM, Novak G, Nagelberg J, Patel M. The role of brain computed tomography in evaluating children with new onset of seizures in the emergency department. Epilepsia 2000;41:950-954

13. Shinnar S, Kang H, Berg AT, Goldensohn ES, Hauser WA, Moshe SL. EEG abnormalities in children with a first unprovoked seizure. Epilepsia 1994;35:471-476.

14. King MA, Newton MR, Jackson GD, Fitt GJ, Mitchell LA, Silvapulle MJ, Berkovic SF. Epileptology of the first-seizure presentation: a clinical, electroencephalographic, and magnetic resonance imaging study of 300 consecutive patients. Lancet 1998;352:1007-1011

15. CharutaJoshi, Wawrykow T, Patrick J, Prasad A. Do clinical variables predict an abnormal EEG in patients with complex febrile seizure? Seizure. 2005;14:429-34.

16. Yücel O, Aka S, Yazicioglu L, Ceran O (2004) Role of early EEG and neuroimaging in determination of prognosis in children with complex febrile seizure. PediatrInt 46:463-467.

17. Benbadis SR, Tatum WO.

Overinterpretation of EEGs and misdiagnosis of epilepsy. J Clin Neurophysiol. 2003;20(1):42-44 